Article

\title{
How Are University Gyms Used by Staff and Students? A Mixed-Method Study Exploring Gym Use, Motivation, and Communication in Three UK Gyms
}

\author{
Frances Rapport ${ }^{1, *}$, Hayley Hutchings ${ }^{2}$, Marcus A. Doel ${ }^{3}$ (i) , Bridget Wells ${ }^{2}$, Clare Clement ${ }^{4}$ (D), \\ Stephen Mellalieu ${ }^{5}$, Sergei Shubin ${ }^{4}$, David Brown ${ }^{5}$, Rebecca Seah ${ }^{1}{ }^{(1)}$, Sarah Wright ${ }^{6}$ and \\ Andrew Sparkes ${ }^{7}$ \\ 1 Australian Institute of Health Innovation, Macquarie University, Sydney, NSW 2109, Australia; \\ Rebecca.seah@mq.edu.au \\ 2 Institute of Life Sciences, College of Medicine, Swansea University, Swansea SA2 8PP, UK; \\ h.a.hutchings@swansea.ac.uk (H.H.); b.wells@swansea.ac.uk (B.W.) \\ 3 Department of Geography, College of Science, Swansea University, Swansea SA2 8PP, UK; \\ m.a.doel@swansea.ac.uk \\ 4 Bristol Medical School, University of Bristol, Bristol BS8 1TH, UK; c.clement@bristol.ac.uk (C.C.); \\ s.v.shubin@swansea.ac.uk (S.S.) \\ 5 Cardiff School of Sport and Health Sciences, Cardiff Metropolitan University, Cardiff CF5 2YB, UK; \\ smellalieu@cardiffmet.ac.uk (S.M.); dbrown@cardiffmet.ac.uk (D.B.) \\ 6 Patient Knows Best, St John's Innovation Centre, Cambridge CB4 0W, UK; Sarah@patientsknowbest.com \\ 7 Carnegie School of Sport, Leeds Becket University, Leeds LS1 3HE, UK; A.C.Sparkes@leedsbeckett.ac.uk \\ * Correspondence: Frances.rapport@mq.edu.au; Tel.: +61-2-9850-2320
}

Received: 4 December 2017; Accepted: 18 February 2018; Published: 1 March 2018

\begin{abstract}
This study examined university gym use by staff and students using mixed methods: participant observation and an e-survey. Research in three UK universities entailed 16 observation sessions and an e-survey that reached 3396 students and staff. The research focused on gym use, the gym environment, the presentation of the self, and social interaction within gym spaces. The gyms were found to have a difficult role to play in providing functionality for some, while helping others to be active and minimize feelings of isolation and lack of control. This led to these gyms developing spaces of exercise rather than therapeutic spaces, and divisions in use of space, with some areas rarely used and often highly gendered, resulting in contested meanings produced within Healthy University discourses and physical activities.
\end{abstract}

Keywords: gym use; gym culture; university staff; university students; higher education

\section{Introduction}

UK adults spend up to $60 \%$ of their waking hours in the workplace [1], providing an opportune micro-environment for cost-effective health-based interventions [2] that aim to reduce ill health in the workforce and the populace [3]. With over two million students and a third of a million staff, the UK's 169 Higher Education Institutions, most of which are universities, are ripe for such interventions. Indeed, the Higher Education Funding Council for England (HEFCE) established a "UK National Healthy Universities Network" in 2006 to foster a "whole university" approach to making "the places within which people learn, live, work and play supportive to health and wellbeing" [4]. In such a context, university gyms take on heightened significance, as places for the training and exercise of individual bodies for particular ends (e.g., functional fitness, sporting prowess, body building, and 
body sculpting), as well as places for cultivating the health and wellbeing of the university population as a whole (e.g., rates of sickness absence, occupational stress, and absenteeism). They are recast according to the double articulation of what Foucault (2003) famously called "disciplinary power" and "bio-power" "after a first seizure of power over the body in an individualizing mode, we have a second seizure of power that is not individualizing but, if you like, massifying, that is directed not at man-as-body but at man-as-species" (Foucault 2003, p. 243) [5]. This double articulation "molds" individual bodies while "modulating" collective life [6]. The very notion of a "Healthy University" gives spatial expression to such a double articulation of disciplinary power and bio-power; of molding and modulation.

While research exists on the motivation for gym participation and culture in general populations [7], the evidence base with respect to university students and staff is limited. Students are an under-researched population, despite the fact that universities are known to shape life-long health behaviors [8] and a quarter gain significant weight in their first semester [9]. Understanding the motives for and patterns of students' gym use may allow better public health interventions [10]. Similarly, university staff are an under-researched population, and the evidence base has barely surpassed the level of truism: staff are motivated to engage in physical activity by factors such as a concern for their health and family, but time constraints and work commitments are major barriers to actual participation [11].

In 2010, fitness gyms were reported to be the most popular "sporting activity" among adults in England [12], offering a number of exercise opportunities including cardiovascular, weight resistance, and free weights. Unlike most fitness classes, gyms typically comprise mixed-gender settings, and yet the use of gym equipment and space is typically male dominated [13]. Indeed, gender is salient in gym environments with particular relevance to social comparison, culture, and social support [14]. The physical structure of gyms may also contribute to gendered spaces [15] and lead to inequity in usage [16].

Gym environments also offer the opportunity for individuals to "care for the self" well beyond the limited purview of health and wellbeing by cultivating cultural capital, symbolic value, and embodied states [17] suitably tailored to modern consumer markets [18]. In the social space of the gym, the toned, ordered and visible body is the desired form of physical capital, and the gym presents the principal space where the quest for, and attainment of, physical capital takes place. In gyms containing mixed users, however, conflicts in how this capital should be constructed and achieved may be likely [19]. Gyms can also represent a meeting place and sustain a sense of community, including belonging and emotional connection [20].

Gyms, therefore, according to Sparkes (2015), are places where people can experience and come to understand themselves in very different ways. Sparkes illustrates how, in the gym, the same person can very quickly feel the burning sensation of shame and the sublime flow of intense embodiment [21]. It can be heaven (liberating), hell (repressive), or somewhere in between at any given moment. While as a territory it may have fixed features, the landscape within it is fluid and dynamic [21], with multiple bodies coming into being in various combinations, some of which are "individuated" bodies (i.e., composed as individual), but all of which are irreducibly "collective" bodies (i.e., every "body" is composed, fabricated, and assembled). Deleuze and Guattari (1987) might describe it as an "assemblage" in a perpetual process of becoming, which is composed of heterogeneous components that enter into relations of attraction and repulsion with one another [22]. There are physical objects, happenings, and events, but also regimes of signs, utterances, and so on. The crucial point is that such an "assemblage" works with bodies, through bodies, and on bodies to mold and modulate both individuals and life itself [23]. It is within the gym as a territory, a landscape, and an assemblage that peoples' understanding of themselves as exercising beings (or exercised becomings) unfolds.

Here, they become situated and aware of themselves (e.g., as aged and gendered) and as both a public body-object and a private subject-body in relation to other gym users via the practices of doing and performing their bodies [24]. The experience of embodiment is not a purely private affair, but is mediated by continual interactions with other people and objects. As Blackman (2008) suggests, 
our bodies in the gym should not be conceived of as bounded and singular, but as open systems that "connect to others, human and non-human, so that they are always unfinished and in a process of becoming" (Blackman, 2008: p. 105) [25]. She talks of multiplicity, movement, articulation, process, and enactment for understanding the production of bodies across different sites, locations, and practices. When exploring the role of a facility in shaping health behaviors it is, therefore, important not only to comprehend motives and patterns of exercise activity, but also to observe how people present themselves in relation to one another in the gym environment. Equally, due attention should be given to how the physical geography of such surroundings shapes these relationships and behaviors over time.

The paper's purpose is to advance the evidence base that exists which determines the experiences and motivations of staff and students in higher education for using university gyms. While we know a considerable amount about the general population's experiences and motivations, how bodies perform and people present themselves in gyms, by specifically considering university gyms as the locus of activity, we aim to clarify staff and students' behaviors, motivations, and relationships in these exemplary sites of molding and modulation, where disciplinary power and bio-power are doubly articulated. Accordingly, the research aimed to investigate how staff and students use university gyms, including their relationships with the spaces of the gyms and with each other, and their motivations for using these gyms and any impediments to such use. The university gym, while perhaps identical in each and every respect to gyms outwith the university sphere (thus totally isomorphic), aims not simply to mold particular bodies (physically, socially, culturally, morally, politically, etc.) but to modulate the university population (as a whole which it should be noted is heavily skewed towards the 18-25 age group, a key difference from most commercial gyms): its health, its wellbeing, its sickness rates, its productivity, its creativity, etc.- -a kind of bio-power [26] and thus is uniquely different in this respect. In that sense, the study explores the "Healthy University" initiative as a biopolitical notion par excellence, thus attempting to unpack the apparatuses of modulation and not just molding of individual bodies.

\section{Methods}

To pursue our aims, we adopted a mixed-method approach. Participant observations were undertaken in the gyms of three UK universities (based in England and Wales, and referred to as University A, B, and C hereafter). These universities were chosen to include modern, teaching-intensive universities (Universities A and B), and a more traditional, research-intensive institution (University C), and to cover gym facilities of varying sizes and arrangements, with different procedures for use, and catering to a wide range of users, including recreational, training, and teaching. We also undertook an e-survey that was open to all staff and students within the three universities, thus enabling us to situate the data for gym users within a broader university culture. The study received ethical approval from the lead University's Research Ethics Committee.

\subsection{Participant Observation}

Five researchers, all with previous experience of gym use and of observation data capture and analysis, undertook sixteen observations in total at the three university gyms, each of which lasted from thirty to sixty minutes. Observations were undertaken at different times of the day, on weekdays and weekends, in different parts of the gym, exercising alongside staff, students, and other users, to ensure as wide a range of situations were included as possible. The observations were conducted to conform with the simple observation method described by Bryman (2012) in his writing on unobtrusive methods in social research [27], emphasizing the need to concentrate on explicit and present observations, executed in situ, according to: (1) exterior physical signs, (2) expressive movement (3) physical location (4) in situ conversation and (5) behavior associated with time, rather than experimental contexts that involve coding and more systematic assessment. In addition, our observations were conducted to conform with the theoretical notions we have already mentioned in this paper of a "Healthy University", aiming to emphasize body modulation, rather than bodily change, in order to conform 
to a certain way of being (molding the body to fit the Healthy University ethos). As a result, our observers, (and this was picked up again in a survey described below), were instructed to consider not only the equipment that was used, efforts that were made and the spaces that were filled at any one time, but also relationships that were in evidence, the sense of belonging expressed and shows of unity on offer, as well as how people identified with the space.

In this study, observers considered individually what was occurring in each different gym setting. Individual considerations were then discussed among the group of observers as part of ongoing workshops and group assessments, with which this research team is well versed (see [28,29]), and notes were compared and contrasted, to derive a final consensus opinion across the group of observers, to ensure rigor in the method, and to include the full range of observations. Notes were written up following each observation and a set of discussions took place between observers and senior qualitative methodologists (FR, MD, AS) according to the following: physical aspects of the gym, including its location and material characteristics; spatial aspects, including layout and equipment distribution; the number, type, and characteristics of gym users; their use of the gym, its spaces, and its equipment; their relationship to and interaction with each other; their movement around the gym; and the researchers' experience of observing others in the gym [30]. Observations were aggregated from all three sites, with similarities and differences noted. Preliminary analysis was undertaken to inform the development of the e-survey. This approach to mixed methods, using a sequential rather than a concurrent strategy, allows one kind of data (in this case qualitative data) to inform the way the next kind of data (in this case quantitative data) will be collected, and to enable the secondary data capture to expand on aspects of the primary data capture, but employing a radically different perspective. It is a technique that is easier to implement than a concurrent mixed method design because steps fall into clearly different stages, and though more time is needed for data capture overall, it ensures a thorough investigation of the topic in question [31].

\subsection{E-Survey}

The e-survey was carried out at all three universities between May 2014 and December 2014, using a web-based platform that gave respondents easy access to the e-survey from a variety of devices. Demographic information was collected from all respondents, and those who had used a university gym within the previous year were questioned about: times of use; equipment use; reasons for use; their routines; their gym experiences; their engagement with others; and their impressions of the gym. Most of the questions were answered using sets of pre-determined options that were developed from our participant observation work, and respondents had the opportunity to provide free-text comments to provide additional information.

\subsection{E-survey Data Analysis}

All data were entered and stored onto an SPSS (v21) database. The survey data are presented as descriptive data in the form of frequencies and percentages. Where statistical comparisons were made, the Chi-squared test for categorical data was used. A $p$-value of less than 0.05 was regarded as significant. Analysis was only undertaken where respondents had indicated that they had used the University gym within the last 12 months.

\section{Results}

The results of the e-survey are presented below in narrative and tabular formats, followed by the three overarching themes emerging from the analysis of the participant observations and the results of the e-survey: (1) Environment; (2) Communication and engagement; and (3) Participants' relationship with the gym. 


\subsection{E-Survey Participants}

The number of university students, staff, and their family members with membership at the gyms of the three universities are shown in Table 1.3396 people took part in the e-survey. Of the respondents $996(29.3 \%)$ indicated that they had used the gym within the last 12 months. The analysis therefore focuses on these 996. The distribution across the three universities and the three types of gym users is shown in Table 2; while their age profile is shown in Table 3; and their ethnicity and gender is shown in Table 4.

Table 1. Number of university students, staff, and family members at each gym.

\begin{tabular}{ccc}
\hline University A & University B & University C \\
\hline Students: $\mathbf{9 7 5}$ & Students: 3250 & Students: 2890 \\
Staff: 282 & Staff: 574 & Staff: 273 \\
Family of staff: N/A & Family of staff: $\mathbf{2 5 9}$ & Family of staff: $\mathbf{2 0 1}$ \\
\hline
\end{tabular}

Table 2. E-survey respondents according to university and status.

\begin{tabular}{ccccc}
\hline & University A & University B & University C & Total \\
\hline & $(n=914)$ & $(n=727)$ & $(n=1755)$ & $(n=3396)$ \\
\hline Students & $668(73.1 \%)$ & $416(57.2 \%)$ & $1251(71.3 \%)$ & $2335(68.8 \%)$ \\
Staff & $198(21.7 \%)$ & $258(35.5 \%)$ & $424(24.2 \%)$ & $880(25.9 \%)$ \\
Dual $^{\dagger}$ & $43(4.7 \%)$ & $48(6.6 \%)$ & $58(3.3 \%)$ & $149(4.4 \%)$ \\
Others & $5(0.6 \%)$ & $5(0.7 \%)$ & $22(1.3 \%)$ & $32(0.9 \%)$ \\
\hline
\end{tabular}

Note: ${ }^{\dagger}$ These respondents are both members of staff and students of a university.

Table 3. Age profile of e-survey respondents.

\begin{tabular}{ccccccc}
\hline & $\mathbf{1 8 - 2 4}$ yrs & $\mathbf{2 5 - 3 4}$ yrs & $\mathbf{3 5 - 4 4}$ yrs & $\mathbf{4 5 - 5 4}$ yrs & $\mathbf{5 5 - 6 4}$ yrs & $\mathbf{6 5 +}$ yrs \\
\hline Percentage & $58.2 \%$ & $19 \%$ & $11.3 \%$ & $7.7 \%$ & $3.2 \%$ & $0.4 \%$ \\
\hline
\end{tabular}

Table 4. Ethnicity and gender of e-survey respondents.

\begin{tabular}{cccc}
\hline White & Non-White & Females & Males \\
\hline $2942(86.6 \%)$ & $454(13.4 \%)$ & $2071(60.9 \%)$ & $1325(39.1 \%)$ \\
\hline
\end{tabular}

\subsection{E-Survey Participants Participants Who Had Used the University Gym in the Last 12 Months}

Of the 996 e-survey respondents who had used the gym in the last 12 months, $46.5 \%$ were male and $53.55 \%$ were female. These users are the focus of this paper. $575(57.7 \%)$ used the university gym regularly (either several times a week $(402 ; 40.4 \%)$ or weekly $(173 ; 17.4 \%)$ ). There was no difference between males and females with regards to frequency of use between regular and non-regular users (less than weekly) with $57.7 \%$ of males vs. $57.8 \%$ of females regularly attending the gym ( $p=0.97$ ). There was also no difference between staff only vs. student only users with respect to frequent vs. infrequent use ( $p=0.98$ ) with $58.1 \%$ of staff vs. $58.1 \%$ of students regularly using the gym. The most popular time for attending the gym was the afternoon or evening, during the week, rather than at the weekend. "Fitness" was cited as the overwhelming reason for gym use (cited by $77.1 \%$ of males and $81.8 \%$ of females). This was not statistically different between males and females ( $p=0.07)$. The five other reasons were also very important: muscle work (males $67.6 \%$ vs. females $27.4 \% ; p=0.000$ ); health (males $40 \%$, females $45.8 \%$; $p=0.64$ ); weight loss (males $31.1 \%$, females $52.3 \%$; $p=0.000$ ); training (males $46.9 \%$, females $27.6 \% ; p=0.000$ ) and relaxation (males $23.1 \%$, females $22.7 \%$; $p=0.88$; Table 5 ). 
Table 5. Reasons $\ddagger$ for gym use $(\mathrm{N}=3396)$.

\begin{tabular}{cc}
\hline Reason & Number (\%) \\
\hline Fitness & $506(14.9 \%)$ \\
Training & $365(10.7 \%)$ \\
Muscle work & $460(13.5 \%)$ \\
Weight loss & $425(12.5 \%)$ \\
Social & $106(3.1 \%)$ \\
Relaxation & $229(6.7 \%)$ \\
Health & $430(12.7 \%)$ \\
Other & $21(0.6 \%)$
\end{tabular}

Note: $\ddagger$ Respondents could choose more than one reason. Percentages are calculated as the number of people specifying each of the reasons divided by the total respondents to the e-survey.

Aerobic equipment was used frequently by both males and females ( $67 \%$ males vs $86 \%$ females; $p=0.000)$. Males also cited weights machines (72.1\%) and free weights $(69.5 \%)$ as being frequently used. This contrasted with the female respondents who used weights machines $(47.3 \% ; p=0.000)$ and free weights $(34.3 \% ; p=0.000)$ less frequently. Females used mats more frequently than males (males $41.9 \%$, females $45.4 \% ; p=0.27$ ), although this was not statistically significant. Students and staff both cited aerobic equipment as the most frequently used (staff $81.8 \%$ vs. students $75 \% ; p=0.03$ ). Weight machines (staff $52.5 \%$, students $61.1 \% ; p=0.020$ ), free weights (staff $38.6 \%$, students $55.1 \%$; $p=0.000$ ), and mats/wall bars (staff $39.8 \%$, students $45.3 \% ; p=0.14$ ) were cited as the next three most commonly used categories. There was a difference between staff and students with respect to usage of aerobic, weight machines and free weights. Only a small proportion did not follow a set routine (males $8.9 \%$, females $17.3 \% ; p=0.000$ ), while $27.7 \%$ always followed a set routine (males $36.1 \%$ vs. females $20.5 \% ; p=0.000$ ) - with a further $52.3 \%$ "sometimes" following a set routine (males $50.1 \%$ vs. females $54.2 \% ; p=0.20$ ). Similarly, a large number of staff and students said that they always followed a routine (staff $28.4 \%$. students $28 \%$; $p=0.91$ ) or sometime followed a routine (staff $51.7 \%$, students $52.7 \%$; $p=0.79$ ), with very few saying they had no routine (staff $11.9 \%$, students $13.7 \% ; p=0.47$ ). There were no differences between staff and students with regards to routine. Most found it "easy" (63.8\%), to fit gym use into their work-life balance (males $64.8 \%$, females $62.9 \%$; $p=0.53$ ). Staff and students also both indicated that it was easy to fit the gym into their work-life balance (staff $61.4 \%$, students $65.1 \% ; p=0.31$ ) Only $14 \%$ regarded using the gym as a "luxury" (males $14.9 \%$, females $13.1 \% ; p=0.42$ ). $44 \%$ found the gym a "convenience" (males $40.2 \%$, females $47.3 \% ; p=0.02$ ), $31.8 \%$ a "necessity" (males $33.5 \%$, females $30.4 \%$ females; $p=0.33$ ), and $21 \%$ a "priority" (males $26.6 \%$, females $16.3 \% ; p=0.000$ ). $10.6 \%$ of staff documented that the gym was a luxury compared with $14.9 \%$ of students $(p=0.10)$; a convenience (staff $48.3 \%$, students $42.7 \% ; p=0.14$ ), a necessity (staff $28.4 \%$, students $33 \% ; p=0.19$ ) and a priority (staff $18.6 \%$, students $22.1 \% ; p=0.26$ ).

When asked about recalling a time when they were aware of their body in the gym, $228(22.9 \%)$ recalled "feeling good" (males 26.8\%, females 19.5\%; $p=0.006$ ) about their body, $145(14.6 \%)$ recalled "feeling bad" (males 11.7\%, females 17.1\%; $p=0.02$ ) about their body, and $213(21.4 \%)$ recalled feeling neither good nor bad (males 21.3\%, females 21.4\%; $p=0.99$ ). Differences between staff and students were good time (staff $16.9 \%$, students $25.9 \% ; p=0.005$ ), bad time (staff $17.4 \%$, students $13.6 \% ; p=0.15$ ), and neither good nor bad (staff $19.1 \%$, students $22.6 \% ; p=0.26$ ). A large proportion of people indicated that they did not speak to anyone while they were in the gym (males $22.2 \%$, females $23.1 \% ; p=0.76$; staff $22.9 \%$, students $21.9 \%$; $p=0.74$ ), some talked to gym staff (males $23.3 \%$, females $27.8 \%$; $p=0.11$; staff $46.6 \%$, students $18.4 \% ; p=0.000$ ); to friends (males $65.2 \%$, females $57 \% ; p=0.008$; staff $30.9 \%$, students $72.7 \% ; p=0.012$ ); to colleagues (males $24.2 \%$, females $16.5 \% ; p=0.003$; staff $50.8 \%$, students $8.9 \% ; p=0.000$ ); and to trainers (males $7.1 \%$, females $6.2 \% ; p=0.55$; staff $7.6 \%$, students $6 \% ; p=0.38$ ). Forms of non-verbal communication included: exercising alongside someone they knew (males 51.2\%, females $50.1 \% ; p=0.73$; staff $28.4 \%$, students $59.6 \%$; $p=0.000$ ); observing other gym users (males $49.5 \%$, 
females $44.8 \% ; p=0.15$; staff $44.9 \%$, students $48.1 \%$; $p=0.39$ ); and observing trainers (males $13.6 \%$, females $12.2 \% ; p=0.51$; staff $14 \%$, students $12.9 \%$; $p=0.66$ ).

Just under half of gym users said the gym was "equivalent" to others they had used (males $40.6 \%$, females $40 \%$; $p=0.70$; staff $43.2 \%$, students $40.1 \%$; $p=0.41 \%$ ), while a slightly smaller number said that it was not as good (males $36.5 \%$, females $40 \% ; p=0.26$; staff $34.3 \%$, students $38.3 \%$; $p=0.28$ ). Reasons for using the university gym in preference to other gyms included: convenience (males $80.8 \%$, females $77.3 \% ; p=0.18$; staff $84.7 \%$, students $76.6 \%$; $p=0.008$ ); local (males $52.3 \%$, females $52.9 \%$; $p=0.84$; staff $43.2 \%$, students $57.1 \%$; $p=0.000$ ); cheap (males $51.6 \%$, females $53.8 \%$; $p=0.48$; staff $70.8 \%$, students $45.6 \%$; $p=0.000$ ); used by people they knew (males $27 \%$, females $27.2 \%$; $p=0.94$; staff $13.6 \%$, students $33 \%$; $p=0.000$ ); liked the equipment (males $25.5 \%$, females $13.1 \%$; $p=0.000$; staff $17.4 \%$, students $20.1 \% ; p=0.35$ ); and felt like a comfortable place (males $16.6 \%$, females $18.6 \% ; p=0.42$; staff $22 \%$, students $16.4 \% ; p=0.052$ ).

University gym users characterized a "good session" as: having a good workout (males $84 \%$, females $79.5 \% ; p=0.07$; staff $76.7 \%$, students $83.7 \% ; p=0.015$ ); getting fitter and healthier (males $58.1 \%$, females $66.8 \% ; p=0.005$; staff $67.8 \%$, students $61.3 \% ; p=0.073$ ); doing what was needed (males $48.8 \%$, females $40 \% ; p=0.003$; staff $42.4 \%$, students $44.7 \%$; $p=0.53$ ); letting off steam (males $41.5 \%$, females $38.1 \% ; p=0.28$; staff $30.5 \%$, students $42.6 \% ; p=0.001$ ); losing weight and/or burning calories (males $27 \%$, females $47.1 \%$; $p=0.000$; staff $39 \%$, students $38.4 \%$; $p=0.88$ ); getting an "exercise high" (males $34.1 \%$, females $28.7 \%$; $p=0.07$; staff $26.7 \%$, students $33 \% ; p=0.07$ ). They characterized a "bad session" as: not having a good workout (males $73.9 \%$, females $61.4 \% ; p=0.000$; staff $59.3 \%$, students $70.6 \%$; $p=0.001$ ); not doing what was needed (males $51.4 \%$, females $46.5 \%$; $p=0.13$; staff $41.9 \%$, students $51.7 \% ; p=0.009$ ); not getting fitter or healthier (males $42.1 \%$, females $48 \%$; $p=0.06$; staff $40.3 \%$, students $47.4 \% ; p=0.06$ ); not losing weight and/or burning calories males $26.7 \%$, females $35.5 \% ; p=0.000$; staff $24.2 \%$, students $31.9 \%$; $p=0.025$ ); not letting off steam (males 30\%, females $22.3 \%$; $p=0.009$; staff $13.1 \%$, students $30 \% ; p=0.000$ ); and not getting an "exercise high" (males 22.9\%, females 21\%; $p=0.47$; staff $14.8 \%$, students $24.4 \% ; p=0.002$ ).

Most university gym users felt that using the gym improved their: physical wellbeing (males $89.6 \%$, females $84.8 \% ; p=0.024$; staff $90.3 \%$, students $86.6 \% ; p=0.14$ ); mental wellbeing (males $87.7 \%$, females $80 \% ; p=0.001$; staff $87.3 \%$, students $79.4 \% ; p=0.007$ ); and self-image (males $75.4 \%$, females $67.2 \% ; p=0.004$; staff $67.4 \%$, students $73.3 \% ; p=0.08)$. Most felt comfortable using aerobic equipment (males $81.2 \%$, females $86.9 \% ; p=0.015$; staff $85.6 \%$, students $84.1 \% ; p=0.59$ ). Fewer, however, felt comfortable using weights machines (males $77.8 \%$, females $40.7 \%$; $p=0.000$; staff $55.1 \%$, students $58.9 \%$; $p=0.31$ ); mats and/or wall bars (males $53.1 \%$, females $42 \%$; $p=0.000$; staff $45.3 \%$, students $48.1 \%$; $p=0.46$ ); or free weights (males $66.3 \%$, females $25 \% ; p=0.000$; staff $37.7 \%$, students $46.4 \%$; $p=0.02$ ).

\subsection{Overarching Themes}

Three overarching themes emerged from the data analysis. The first and second themes relate to the participant observations. The third relates to the results of the e-survey, particularly regarding respondents' free-text comments.

Theme 1: Environment

All three gyms were designed as part of the wider campus layout, including its sport facilities, and were accessible to both staff and students. However, their external arrangements varied. In University B for example, gym facilities were split across two buildings, one containing the gym and the other free weights, lifting bars and platforms, and numerous dumbbells. At University C, only 12 people could train in the free weights area at any one time, and this may have affected observations that it was a space for "serious trainers" interested primarily in body building. Inside all three gyms, equipment and gym spaces were strategically divided into different sections or training zones, with distinct sets of users observed in each. This was heavily gendered, and, to some extent, age-dependent. In University A, women of all ages and older men predominated in the running machine, step machine, and cycle 
machine zones, with younger women making more effort, and using more difficult machines, than older women. Younger men were less likely to exercise in these zones, and this was particularly noticeable in the "serious trainer" gym of University C. Furthermore, when they did, exercise duration was relatively short. On these occasions, younger men used the running machines for higher intensity training or as a warm-up, prior to moving on to the weights machines or free weights areas. In the free weights areas, dumb bells and bar bells were in proliferation, and the younger men tended to gravitate quickly to these areas, though some female track athletes were also observed there. However, even in this "serious trainer" gym, the use by female track athletes was markedly different to the use by younger men. In corroboration with the e-survey results, women used free weights for functional fitness purposes, whereas men used free weights for a mixture of functional fitness and body building. Women were also more likely to keep to themselves, and appeared less confident than men during workout regimes. Men looked at themselves quite openly, and at others, in the large floor-to-ceiling mirrors, and, on occasion, worked out in pairs. It was unusual for women and men to work out together in any area.

\section{Theme 2: Communication and Engagement}

Most gym users got on quietly with what they were doing through independent training, without much social interaction, other than occasionally turning to someone for support in using gym equipment appropriately for building up of certain muscle groups (predominantly males). There was a clear sense of solitary activity, to enhance one's own body and fitness. Individuals also occasionally helped each other hold weights or put down a heavy weight. Lack of interaction was evident across all three gyms, despite the noticeable presence of very mixed user groups throughout. One gym (University A) had a multiplicity of social groups, including minority ethnic students and international students at busy times, such as the late afternoon. However, there was often a level of segregation between groups, and a lack of social interaction, that was fostered to a large extent by the gym layout and placement of equipment and very different uses of the gym by its methods.

Gym instructors were noticeably absent at most times of the day in all gyms, with the exception of the early evening "rush-hour". At this time, equipment, particularly the running and cycling machines, was at a premium. When, on occasion, a gym instructor was observed, their presence along with the type of music being played affected others' demeanor and voice, and enlivened the workout areas. Users tended to be more numerous and louder. Groups of users coalesced around the instructors, and others outside the group had to wait for a slot to use certain equipment. These rare moments affected a flow of individuals around the equipment. Users' gazes became more intense, and workouts were more introverted.

Linked to this, a very high number of individuals wore headphones at busy times of the day, and used equipment to block out others' noise and create a barrier between people who were unwilling to join in. At these times too, smart phones, and other mobile devices, were particularly noticeable, allowing people to take legitimate "rests" from the fairly consistent rhythm of the gym. Smart phones were used to: listen to music, login to "Apps", text, or make phone calls. Given that these people were still communicating with one another, it was unclear whether they were also listening to music or blocking out some background noise.

Lack of clear communication among users, other than at unusually busy times, meant that the rhythm of the workout routine usually went unhindered. There was an overarching focus on "work being done" (expressed in terms of productivity rate), and an adherence to function over the intention to maintain a sense of community (often evident in non-university gyms) through social communication. This illustrates Foucault's idea of bio-power we introduced earlier in as much as the university gym modulates the collective life (the staff and student population) through governing possibilities for action. As will become clear in the discussion, lack of engagement was the norm, leading to expectations of the importance of individualized behavior. 
In all gyms, while some sense of community was developed around male camaraderie at particularly busy times of the day, women tended to avoid eye contact and interaction. Small male partnership groups illustrated this sense of camaraderie, with pairs or "threes" being quite common in the free-weights area. It was here that men appeared confident, while women did not look confidently around the gym or at one another.

Theme 3: Participants' Relationship with the Gym

Most gym users appeared familiar with their surroundings and confident in their approach to using the space and its equipment. In accordance with this, the e-survey found most respondents followed a set routine, either sometimes or all the time. On several occasions people new to the gym entered looking unsure, especially when it was difficult to locate a member of staff to speak to before trying out gym equipment.

Most respondents to the e-survey recalled good, rather than bad, experiences, but bad experiences included being aware of being "unfit" or "out of shape", and feeling "too fat" or "too thin". For example: "Feeling skinny and lacking in muscle-build"; "I have a lot of body image issues, I am not a fit or a slim person"; "I'm obese, old and unfit". It was clear from many comments that some people had an uneasy relationship with the gym, finding it to be a place where negative perceptions of body image come to the surface, reinforced by others' observations. Some people talked negatively about their bodies compared to younger, fitter users. Many felt self-conscious, compounded by the prevalence of mirrors: "Mirrors everywhere. I am tall and thin and this shows compared to others"; "Loss of confidence looking at my body against younger, fitter one".

While community sports centers were designed with the philosophy of sports for all, university gyms were often designed to support sporting excellence often defined in bio-political terms [26] within the statistical order of efficiency, productivity and achievement. Responses from our e-survey showed that more casual gym users felt uncomfortable training alongside more serious sports people: "Using equipment alongside professional sports people is depressing when you are a middle-aged woman"; "A lot of sports teams use the gym, and being a nerd I can get self-conscious". Perhaps related to this, the cultural phenomenon of "posturing" was observed, particularly in the weights areas. Men postured in front of mirrors leaving women feeling inhibited or put off from those areas: "I am always aware of my body and other people's bodies, and posturing". Several other e-survey comments supported the observations that the gyms were gendered spaces, with informal "male zones" being particularly noticeable: "A small gym full of men is very intimidating as a casual female user"; "Boys staring when in the weight machine area made me feel self-conscious"; and "I remember one of the first times using the free weights ... I was acutely aware that I was the only female body in that area of the gym and I felt quite intimidated".

Some respondents talked about their desire to improve their health-related experiences, and by so doing improve their body image, such as gaining better body tone: "I was surprised by how much larger and well-defined my shoulders had become"; "I've dropped a few dress sizes". Many people described the journey from being unfit and "out of shape" to becoming fitter as very life-affirming: "Initially felt bad due to being overweight. After a while felt good as I could feel my body getting stronger". Those who saw themselves as "out of shape" were even less likely to move confidently around different areas and, as a result, this theme emphasizes a strong link between appearance and confidence. Men, in particular, described themselves in body-image terms.

\section{Discussion}

This study sought to add to the existing evidence base regarding the experiences and motivations of staff and students in higher education for using university gyms. Specifically, through the lens of the "Healthy University" initiative and how staff and students" behaviors, motivations, and relationships exhibited in these exemplary sites of molding and modulation, where disciplinary power and bio-power are doubly articulated. 
The findings from the e-survey responses are consistent with the limited literature surrounding motivation for university gym use of staff and students in higher education [11] in that fitness was the most frequently cited reason for gym use for male and female members alike. The survey provided further fine-grained understanding of motives to exercise by highlighting differences in the forms of fitness engaged in, with males indicating using the gym more for muscle work, while females reported engaging more for reasons associated with weight loss. Insight was also provided into differences in the type of equipment utilized with females reporting feeling less comfortable using weights machines or free weights than their male counterparts.

While these findings are consistent with the extant literature regarding motives and behaviors of gym use in general populations (e.g., $[14,16])$, the open-ended responses from the e-survey together with the participant observations, also provided deeper and novel insight into higher education specific motives and behaviors, and the notion of a "Healthy University" and the subsequent relationships exhibited with the specific university gym environments. Like Klein's (1993) analysis of body-building gyms [32], the aspects discussed here are common issues that concern: (1) the university gym as a multi-use space; (2) the (gendered) division of gym space; and (3) the gym's socio-spatial interactions.

\subsection{The University Gym as a Multi-Use Space: From Fitness to Therapy}

Firstly, our data reinforce the idea that the gym is "a complex social and cultural construction" (Andrews et al., 2005: p. 888) [7]. These respondents confirm Crossley's (2006) view that reasons for using the gym consist of a diverse "vocabulary of motives" in which motivations are learnt, storied, and exchanged [33]. Most respondents reported using the gym for fitness and training, while weight loss and relaxation were also important. This corresponds with Monaghan's (2001) four gym-use outcomes of: healthy body, fit body, youthful body, and attractive body-although we would add a fifth: the "performing body" (focused on functional training for sports performance) [34]. Gym use motivation seems to range from being part of a focused "self-improvement project" [35] to just "feeling good" and developing a better functioning body [36], which accords with Foucault's (2003) double articulation of "individuating" disciplinary power and "massifying" bio-power [5], and Deleuze's (1992) double articulation of "molding" a body and "modulating" a life [6]. To take care of one's self, and to be concerned with one's self, is to transform one's self into a matter of concern-both for oneself and for others. Within the gym, the embodied self is rendered problematic. It poses itself as a problem-whose solution is literally worked out.

The diversity of desired outcomes also connects with Andrews et al.'s (2005: p. 889) suggestion that the view of gyms as "therapeutic places deserves more serious consideration" [7]. Therapeutic landscapes are not only related to the quality of the environment but emerge through health-seeking discourses at various levels [37-39]. In that respect, the UK's Healthy University Strategy suggests a broad concept of "healthy behavior". As Dooris et al. (2014) note, the Healthy University approach focuses on creating environments that support health activities, strengthen health potential, and bridge the gap between health professionals, university staff, and university students [40,41]. From this perspective, therapeutic properties cannot be seen as innate qualities of the university gyms but emerge through the disciplinary and bio-political practices [26], behaviors, and processes that enliven them.

The therapeutic function is strongly suggested by the quarter of respondents who used the gym for relaxation and such things as "feeling better", "mental wellbeing", and a "positive effect on mood". It is apparent that the university gym has a difficult role to play in providing the desired functionality for some users, while also helping others to "be active", avoid obesogenic lifestyles, and minimize feelings of isolation and lack of control, which is strongly connected with student's alienation from physical education [42].

As a result, these three university gyms have developed spaces of exercise rather than therapeutic spaces with contested meanings produced within Healthy University discourses and physical activities. Representations and spatial divisions promoting active lifestyles often embed knowledge, signs, and perceptions reflecting sporting ideals of hetero-masculinity which often stand in contrast to female 
practices of sport and physical activity [43]. As a result, these study gyms created a number of barriers for a significant group of potential users to enact healthy lifestyles. Dominant sporting discourses encourage female users to create separate identities of woman and athlete, leading them to focus less on body "work" and more on finding "healthful" spaces in which to take care of their bodies. This may also help to explain an observed awkwardness among many users regarding free movement around the gyms, and why most staff and students who participated in the e-survey (75\%) choose not to use the university gym at all. Finally, the idea that many users are seeking a therapeutic space raises questions over whether "gym" is an appropriate term given its "contestable description" [33] and whether consideration might be given to other terms, such as "health club" or even "health and fitness zone".

\subsection{The (Gendered) Division of Space}

An emerging issue that connected strongly within the above involves the division of use of space, with many people reporting feeling comfortable using: aerobic equipment (91.9\%), weight machines $(64.6 \%)$, and free weights $(49.6 \%)$. Andrews et al. (2005: p. 889) comment that in their study of body-building gyms, there were "micro-geographies, divisions of space according to the body and type of customer", saying "there is a need to consider the micro-geographies and divisions of space within other fitness places" [7]. The division of space found in these three university gyms tends to be constructed between the aerobics, fixed-weight equipment, and the free-weight-training equipment, and in some of these, physically demarcated and separated spaces too. This division of space creates places where many users rarely go, leaving them colonized by frequent users alone, and leading to micro geographies and spatial practices containing significant gender and interaction elements: "Equipment and gym spaces are strategically divided into different sections or training zones, and distinct sets of users were observed in each zone". The gendered dimension to these zones is remarkably similar to the patterns observed in previous studies of gendered lines in exercise environments $[44,45]$. Indeed, Dworkin's (2003) study of gym use in a Canadian higher education gym, noted females engaged more with aerobic fitness exercise whereas males, particularly young males, engaged more with muscle building via free weight resistance exercises [45]. Due to the apparent segregation of the fitness room by gender, the building of muscle for aesthetic purposes was still noticeably absent in the female user group even among those athletes who used free weights most intensively.

The findings surrounding gender from the work of Coen et al., [44] and also Dworkin [45], suggest, as Johnston (1998: p. 250) argues, "[h]istorically, entry by women into gyms-an exclusively male environment-was not easily achieved" [46]. In addition, in the university gyms in our study, it would seem to remain the case for most users that "while both men and women work out in contemporary gyms, popular conceptions of the gym as a masculine institution continue" [47]. It is important, however, to stress that many men in the university gym environment do not simply enact the dominant stereotypes about gendered division of space and go beyond the drive for muscularity to also include the reduction of body fat [48]. In view of this, any changes to university gym environments made in respect of gender would need to avoid the tendency identified by Craig and Liberti (2007: p. 676) to "feminize" the gym according to stereotypical assumptions about feminine forms of gym use: Rather than merely accommodating women's behaviors, organizational processes shaped them. Moreover, the layout and procedures for the use of machines and the speech norms modeled by the staff called on women to enact particular performances of femininity [47].

\subsection{Socio-Spatial Interactions and Practices}

Sassatelli (1999: p. 228) emphasizes the significance of "how gyms are organized and how their internal organization acquires meaning for clients". The varied uses of the university gym, and the division of space to accommodate some of those uses, also lead to a range of established practices and interactions that are important to appreciate if these spaces are to contribute more fully to the 
UK's Healthy University strategy [49]. As Sassatelli (1999: p. 230) puts it, gyms are a "compound and complex environment", an assemblage of heterogeneous components, in which individuals must "shift inwards—into the world of training-and outwards—back to different external realities" [49]. However, shifting inwards and outwards is especially challenging for university gym members because they not only have very different needs, they also occupy very different social positions within the university. This must be considered when interpreting the $23.8 \%$ of respondents who reported not speaking to anyone while in the gym, the $65.3 \%$ who spoke only to friends, the $21.8 \%$ who spoke only to colleagues, and the $49.6 \%$ who reported observing other gym users

While using the gym themselves. The significant issue here is that despite our observations that the cultural norm in these spaces was to focus "inwards" on the training work to be done and limit communication, the users do talk to other people, engage in non-verbal communication, and observe each other. As our observations demonstrate, there is an element of university gym users watching and listening to other people, and synchronizing their workouts, such as following "people using different equipment"; "watching other students using the machines"; and replicating "something I can learn [from]".

In line with the Healthy University agenda, university gyms are expected to offer more than just a place to exercise while at the same time providing opportunities for staff and students to develop social benefits and contribute to university integration [50]. Many of our respondents highlighted such contradictions: "It's also a bit strange being in a gym at work"; "I prefer not to exercise alongside students"; "[I] don't feel comfortable exercising and changing with my students". There are power relations at play that are embedded in the symbolic place of the university gym and impact upon the significance of the gym interactions.

Our observations mirrored those of Sassatelli (1999), in that twin frames of expression are evident; that of training and intervals between training, both of which involve different forms of acceptable expression $[49,50]$. When training, gym users engage inwardly in "postures, glances, facial expressions" which express "that what they are doing is indeed just and only training" (Sassatelli, 1999: 234). For example, gym users in our study commented on ignoring the gym environment ("the university gym plays music/radio and I can't concentrate"), expressing physical strain and even making noises indicating exertion. In the breaks between training episodes gym users have to engage in a more complex set of interactional practices, involving forms of "face work", and in particular glance management that typically attempt to politely ward off any interactions that might prove disruptive to the workout in ways very similar to that which Sassatelli (1999: p. 234) articulates: During relaxation intervals between exercises, which could easily give way to distracting or disruptive episodes, participants' continued self-absorption is typically underlined by an expressionless, absent face [49]. While remaining on their machine or moving toward the next one, clients may glance around focusing on anything in particular, and they assume inexpressive, or even hostile, expressions or cast a distracted smile, avoiding eye contact.

These forms of interaction are often augmented by the use of a variety of artefacts including headphones and music players, smartphones, notebooks to log exercises, and even watching TV screens in the gyms, thereby expressing what Goffman (1971) termed acceptable forms of "civil inattention" [51]. These moments do, however, provide opportunities for the "gaze", when gym users, while resting or moving between exercises; tend to quietly observe others in the gym. Here, "glance management" is engaged in relation to oneself and others (especially via mirrors). In either case, the unwritten rule appears to be that glances must be short in duration and it is during this gaze that the external reality of one's position re-emerges for viewers and the viewed as is evidenced by comments such as "I felt intimidated by slimmer, fitter students"; "Caught self in mirror-wasn't happy with reflection"; "Conscious of other bodies (younger and older) and not being a performance athlete"; "Feel like I'm judged in there"; "Don't want to be seen training by students"; However, the gaze worked in a positive direction too: "stronger than I thought"; "Good as I realized I had weight loss work to do, so was inspirational". Similarly, verbal communications at this point are typically kept to a 
minimum if they are not to prove disruptive. This is particularly apparent in the institutional context where staff and students sometimes engage in discussions about work issues $(24 \%)$ or general chat $(82 \%)$, which shift users outwards between episodes of training. To ignore colleagues or friends in such a scenario may not be acceptable and so the disruption of one's training to perform "face work" acceptably (from an institutional perspective) may be inevitable.

Therefore, this inward and outward shifting of interaction is crucial to the university gym setting and culture. Its users are intrinsically connected, both professionally and personally, and so the external realities of one's environment are potentially more difficult to set aside and bracket than in other gyms where one is merely a "member". Indeed, the proximity of bodies in the university gym space, their relative exposure, and the necessity for a range of interactions may for some staff and students not be commensurate with the idea of a therapeutic space at all, and this may partially explain why relatively few people join the university gym. These are again in strong evidence in the e-survey comments: "Do not want to see my students while I am training-I really like them, but I also like to have a clear boundary between my personal and work life"; "Don't want to work out around people I could potentially know"; "I find it a bit cramped and busy. It's also a bit strange being in a gym at work.

\section{Conclusions}

The paper has highlighted a number of important issues that should be considered to ensure that university gyms are fit for purpose. First, there are limited proportions of staff and students using university gyms, which must be taken seriously, especially given the UK's Healthy University strategy. Second, there is a clear tension between some of the desired uses and the spaces provided, which is exacerbated by the gendered division of space. Third, social relationships and interactions within the gym have an impact on whether people use the gym at all, and, if they do, how they use it. Consequently, researchers need to reassess what activities and interactions within university gyms mean for both staff and students, and how the assemblage of gym users and gym spaces is double articulated by forms of disciplinary power and bio-power that pose the embodied self and Life itself not only as problematic, but as problems that need to be literally worked out.

The above issues also raise a number of quite practical considerations for university gym developers. Given the great diversity of uses of the university gym and the directive of the UK Healthy Universities discourse towards the molding and modulation of health and wellbeing, a physical separation between general health and wellbeing studios and high-performance fitness gyms may be a relevant consideration. Additionally, university gyms might also consider maximizing the off-peak times usage of these spaces by considering special sessions for particular groups who may otherwise be alienated by the experience: for example, women-only weights sessions, instructor-led sessions and beginners-only sessions. Finally, universities might more explicitly promote their gyms as inclusive therapeutic communities for all their members. By paying close attention to the molding and modulation discourses emanated through signage, gym etiquette rules, and more proactive monitoring of interactional behavior by instructors, much could be done to maximize the staff and student experience in this regard.

One of the strengths of our study was the adoption of a mixed method design via participant observation and survey techniques, overcoming many of the limitations of single method approaches. It should be noted, however, that such designs can be constrained in terms of legitimacy and meaning, philosophical underpinnings, and in respect of the actual pragmatics of undertaking such a study [52]. Additional limitations to the current study may also lie in the extent to which our findings are unique to the university staff and student population or the university gym environment itself. Inquiry is therefore needed to further explore the "Healthy University" initiative as a biopolitical notion and unpack the molding of individual bodies and modulation of the university population (as a whole), as unique to a higher education context or prevalent in other educational or public-sector environments. Lastly, although we have further highlighted the gendered division that exists across the use of exercise 
spaces [44] but within a collegiate context, a clear avenue for future research would be to consider how a gender-diverse person and ethnic minority person may experience the gym environment.

Acknowledgments: We acknowledge Swansea University's "Bridging the Gaps" initiative for providing financial support for the research. The funder had no role in study design; collection, analysis, and interpretation of data; writing the article; or the decision to submit the article for publication. We also acknowledge Vicky Davies for administrative assistance, Swansea University's Healthy Universities Committee Chair and gym-user representatives for attending project meetings, and the university gym managers and their colleagues for their assistance.

Author Contributions: F.R. led the study with H.H. She initiated the study idea and won the funding for the study. F.R. also led the team work activities and oversaw qualitative observations and well as leading the qualitative data analysis aspects. F.R. initiated the first draft of the paper and oversaw all other drafts with H.H. H.H. co-led the study with F.R., designed the survey questions with input from B.W. and led the statistical data analysis. H.H. was instrumental in statistical reporting and took a lead position on publication writing, attending to all statistical aspects of each new iteration. B.W. led the data collection for the surveys and observations, was the study researcher and first point of contact, and supported data analysis and writing the first draft. F.R., H.H. and B.W. managed the day-to-day running of the study and F.R. and H.H. oversaw data gathering, data analysis and write up. M.A.D. helped with the study design, especially aspect of spatial assessment and group belonging, and he supported qualitative data analysis and writing up of the qualitative data. He was also fully involved in publication writing and supported each amendment as the paper progressed. C.C. undertook observations and supported the qualitative data analysis. She attended group work sessions and workshops and assisted with publication writing. S.M. supported quantitative data analysis, attended group work and workshop sessions and input into the discussion of data findings, particularly with relevance to Healthy University initiatives and sports health theories. S.S. helped with the study design, especially aspects of spatial arrangement and group belonging. He attended group work and workshop analysis sessions, and was instrumental in discussions on data content and writing aspects of the paper as regards spatial theory. D.B. oversaw survey development and survey data collection in one of the gyms, and undertook some observations, he also attended group work and workshop sessions, input into data analysis and writing particularly with relevance to sports health theories. R.S. oversaw formatting and referencing of the paper, and revisions for each new iteration. She also assisted in collation and managing track-changes and liaising with all authors as new iterations materialized. S.W. undertook observations and supported the qualitative data analysis. She attended analysis group work sessions and workshops and assisted with publication writing. A.S. supported survey data collection in one of the gyms, and qualitative data collection and analysis. He undertook observations in one of the gyms and attended group work and workshops sessions. He supported data analysis and paper writing.

Conflicts of Interest: The authors declare no conflict of interest.

\section{References}

1. Department of Health. Choosing Activity: A Physical Activity Action Plan; Department of Health: London, UK, 2005. Available online: http:/ / webarchive.nationalarchives.gov.uk/20130105001838/http:/ /www.dh. gov.uk/prod_consum_dh/groups/dh_digitalassets/@dh/@en/documents/digitalasset/dh_4105710.pdf (accessed on 4 December 2017).

2. Baicker, K.; Cutler, D.; Song, Z. Workplace wellness programs can generate savings. Health Aff. 2010, $29,304-311$. [CrossRef] [PubMed]

3. Aldana, S.G.; Anderson, D.R.; Adams, T.B.; Whitmer, R.W.; Merrill, R.M.; George, V.; Noyce, J. A Review of the Knowledge Base on Healthy Worksite Culture. J. Occup. Environ. Med. 2012, 54, 414-419. [CrossRef] [PubMed]

4. Healthy Universities. 2017. Available online: http://www.healthyuniversities.ac.uk/healthy-universities/ key-concepts-health-wellbeing-sustainability / (accessed on 4 December 2017).

5. Foucault, M. Society Must Be Defended: Lectures at the College De France 1975-1976; Picador: New York, NY, USA, 2003.

6. Deleuze, G. Postscript on the Societies of Control; The MIT Press: Cambridge, MA, USA, 1992.

7. Andrews, G.; Sudwell, M.; Sparkes, A. Towards a geography of fitness: An ethnographic case study of the gym in British bodybuilding culture. Soc. Sci. Med. 2005, 60, 877-891. [CrossRef] [PubMed]

8. Leslie, E.; Sparling, P.; Owen, N. University campus settings and the promotion of physical activity in young adults: Lessons from research in Australia and the USA. Health Educ. 2001, 101, 116-125. [CrossRef]

9. Wengreen, H.; Moncur, C. Change in diet, physical activity, and body weight among young-adults during the transition from high school to college. Nutr. J. 2009, 8. [CrossRef] [PubMed] 
10. Lovell, G.; Ansari, W.; Parker, J. Perceived exercise benefits and barriers of non-exercising female university students in the United Kingdom. Int. J. Environ. Res. Public Health 2010, 7, 784-798. [CrossRef] [PubMed]

11. George, E.; Rosenkranz, R.; Kolt, G. Male perceptions of physical activity and sedentary behaviour in a university work environment. Med. Sci. Sports Exerc. 2013, 45, 148-158.

12. Department for Culture Media and Sport. Taking Part: The National Survey of Culture, Leisure and Sport; Adult and Child Report 2009/10; Department for Culture Media and Sport: London, UK, 2010. Available online: www.culture.gov.uk/publications/7386.aspx (accessed on 4 December 2017).

13. Gill, D.; Kamphoff, C. Gender in sport and exercise psychology. In Handbook of Gender Research in Psychology; Chrisler, J., McCreary, D., Eds.; Springer: London, UK, 2010.

14. Pridgeon, L.; Grogan, S. Understanding exercise adherence and dropout: An interpretative phenomenological analysis of men and women's accounts of gym attendance and non-attendance. Qual. Res. Sport Exerc. Health 2012, 4, 382-399. [CrossRef]

15. Slater, A.; Tiggemann, M. Gender differences in adolescent sport participation, teasing, self-objectification and body image concerns. J. Adolesc. 2011, 34, 455-463. [CrossRef] [PubMed]

16. Salvatore, J.; Marecek, J. Gender in the gym: Evaluation concerns as barriers to women's weight lifting. Sex Roles 2010, 63, 556-567. [CrossRef]

17. Bourdieu, P. The social space and the genesis of groups. Theory Soc. 1985, 14, 723-744. [CrossRef]

18. Lee, B. Public Culture. Going Public 1993, 5, 165-178.

19. Tulle, E.; Dorrer, N. Back from the brink: Ageing, exercise and health in a small gym. Ageing Soc. 2012, 32, 1106-1127. [CrossRef]

20. Dionigi, R.; Lyons, K. Examining layers of community in leisure contexts: A case analysis of older adults in an exercise intervention. J. Leis. Res. 2010, 42, 317-340. [CrossRef]

21. Sparkes, A. Ageing and embodied masculinities in physical activity settings: From flesh to theory and back again. In Physical Activity and Sport in Later Life: Critical Approaches; Tulle, E., Phoenix, C., Eds.; Palgrave: London, UK, 2015.

22. Deleuze, G. A Thousand Plateaus C and S; University of Minnesota Press: Minneapolis, MN, USA, 1987.

23. Lazzarto, M. Signs and Machines: Capitalism and the Production of Subjectivity; MIT Press: Cambridge, MA, USA, 2014.

24. Mol, A.; Law, J. Embodied action, enacted bodies: The example of hypoglycemia. Body Soc. 2004, 10, 43-62. [CrossRef]

25. Blackman, L. The Body (Key Concepts); Berg Publishers: Oxford, UK, 2008.

26. Foucault, M. The Birth of Biopolitics. Lectures at the Collège de France 1978-1979; Palgrave Macmillan: New York, NY, USA, 2008.

27. Bryman, A. Social Research Methods; OUP: Oxford, UK, 2012.

28. Rapport, F.; Doel, M.; Hutchings, H.; Jones, A.; Culley, L.; Wright, S. Consultation workshops with patients and professionals: Developing a template of patient-centred professionalism in community nursing. J. Res. Nurs. 2014, 19, 146-160. [CrossRef]

29. Hutchings, H.; Rapport, F.; Wright, S.; Doel, M.; Clement, C.; Lewis, K. Nominal Group Technique Consultation of a Pulmonary Rehabilitation Programme [Version 1; Referees: 2 Approved, 1 Approved with Reservations]; F1000Research: London, UK, 2014.

30. Sparkes, A. Ethnography and the senses: Challenges and possibilities. Qual. Res. Sport Exerc. Health 2009, 1, 21-35. [CrossRef]

31. Creswell, J. Research Design: Qualitative, Quantitative, and Mixed Methods Approaches, 4th ed.; SAGE Publications, Inc.: Thousand Oaks, CA, USA, 2014.

32. Klein, A. Little Big Men: Body Building Subculture and Gender Construction; State University of New York Press: Albany, NY, USA, 1993.

33. Crossley, N. In the gym: Motives, meaning and moral careers. Body Soc. 2006, 12, 23-50. [CrossRef]

34. Monaghan, L. Bodybuilding, Drugs and Risk; Routledge: London, UK, 2001.

35. Maguire, S. The personal is professional: Personal trainers as a case study of cultural intermediaries. Int. J. Cult. Stud. 2010, 11, 211-229. [CrossRef]

36. Waring, A. Health club use and 'lifestyle': Exploring the boundaries between work and leisure. Leis. Stud. 2008, 27, 295-309. [CrossRef] 
37. Conradson, D. Experiential economies of stillness: The palace of retreat in contemporary Britain. In Therapeutic Landscapes; Williams, A., Ed.; Ashgate: Aldershot, UK, 2008.

38. Gastaldo, D.; Andrews, G.; Khanlou, N. Therapeutic landscapes of the mind: Theorizing some intersections between health geography, health promotion and immigration studies. Crit. Public Health 2004, 14, 157-176. [CrossRef]

39. Williams, A. Therapeutic Landscapes; Ashgate: Aldershot, UK, 2008.

40. Dooris, M.; Wills, J.; Newton, J. Theorizing healthy settings: A critical discussion with reference to Healthy Universities. Scand. J. Public Health 2014, 42, 7-16. [CrossRef] [PubMed]

41. Holt, M.; Monk, R.; Powell, S.; Dooris, M. Student perceptions of a healthy university. Public Health 2015, 129, 674-683. [CrossRef] [PubMed]

42. Carlson, T. We hate gym: Student alienation from physical education. J. Teach. Phys. Educ. 1995, 14, 467-477. [CrossRef]

43. Messner, M. Taking the Field: Women, Men and Sports; University of Minnesota Press: Minneapolis, MN, USA, 2002.

44. Coen, S.E.; Rosenberg, M.W.; Davidson, J. "It is gym, like g-y-m not J-i-m”: Exploring the role of place in the gendering of physical activity. Soc. Sci. Med. 2018, 196, 29-36. [CrossRef] [PubMed]

45. Dworkin, S. A Woman's place is in the...cardiovascular room? gender relations, the body and the gym. In Athletic Intruders; Bolin, A., Granskog, J., Eds.; State University of New York Press: Albany, MN, USA, 2003.

46. Johnston, L. Reading the sexed bodies and spaces of gyms. In Places Through the Body; Nast, H., Pile, S., Eds.; Routledge: London, UK, 1998.

47. Craig, M.; Liberti, R. 'Cause that is what girls do': The making of a feminized gym. Gend. Soc. 2007, 21, 676-699. [CrossRef]

48. Bergeron, D.; Tylka, T. Support for the uniqueness of body dissatisaction from drive for mascularity among men. Body Image 2007, 4, 288-295. [CrossRef] [PubMed]

49. Sassatelli, R. Fitness gyms and the local organization of experience. In Sociological Research Online; SAGE Publications Ltd.: Thousand Oaks, CA, USA, 1999.

50. Henchy, A. The influence of campus recreation beyond the gym. Recreat. Sports J. 2011, 35, $174-181$. [CrossRef]

51. Goffman, R. Relations in Public: Microstudies of the Public Order; Allen Lane: London, UK, 1971.

52. Sparkes, A.C. Developing mixed methods research in sport and exercise psychology: Critical reflections on five points of controversy. Psychol. Sport Exerc. 2015, 16, 49-59. [CrossRef] 